\title{
Big Image Data Management on Portable Equipment
}

\author{
Liang Ye \\ Department of Computer Science \\ Beijing Foreign Studies University \\ Beijing, China \\ E-mail: liang_ye@sohu.com
}

\begin{abstract}
Big Data is a new label given to a diverse field of data intensive informatics in which the data sets are so large that they become hard to work with effectively. The term has been mainly used in two contexts, firstly as a technological challenge when dealing with data-intensive domains such as geographical information image, high energy physics, astronomy or internet search, and secondly as a sociological problem when data about us is collected and mined by companies such as Facebook, Google, mobile phone companies, retail chains and governments. In this paper we look at this first issue from a new perspective, namely how can the user gain awareness of the personally relevant part big data that is publicly available in the portable equipment. With a lot of traditional applications such as geography information system (GIS) implanted on portable equipment, how to collect, store, process, analyze, and display big image data becomes a hot field. This paper puts forward a display control technique on portable equipment, which is based on measurement of user's location. At the same time, we do serials of experiment on Android platform to validate them.
\end{abstract}

Keywords- big image date; portable equipment; date management.

\section{INTRODUCTION}

The ultimate goal of massive image data organization and management is to provide high speed data services, such as collect, store, analyze, query, display, refresh and network transmission. [1] Therefore, how to build fast and efficient display mechanism is particularly important. In the display process, it needs to solve two important problems: First, single portable equipment can not store big image data. Second, the traditional organization and management methods of massive image data can not meet the screen of portable equipment. [2, 3]

It is well known that portable equipment has smaller screen and low-performance disposing capability comparing with personal computer, however the traditional GIS depended on high demand for memory size, processor speed and other hardware can not be used for portable equipment directly. [4] So the display method for mobile GIS, which realizes combining traditional GIS and portable equipment, needs to be designed to fit for mobile application environment. [5]

GIS is a system, which could process geographical spatial data. Geographical spatial data includes various data types and levels, with a large quantity of geographical information image data. For mobile GIS, it is so huge that even geographical image data of a campus could not be saved in the memory of portable equipment at one time yet. $[6,7]$

Through analyzing users' demands and customs of querying geographical information on portable equipment in mobile computing environment, we can draw a conclusion that people don't want to obtain geographical information once as much as possible, but want to get the useful information observably shown on screen. So the geographical information image data is cut into levels and blocks, and is indexed in serialization order. Every block of a level could be fit for the memory of portable equipment. They are the partition technique and index technique for mobile GIS. But in order to save the limited memory, it is not enough to only have partition technique and index technique. Because the memory of portable equipment is divided into two parts, one for calculation, and the other for show on the screen. The techniques could only save the memory for calculation and reduce the quantity of querying result data. So in order to save display memory, we put forward the display control algorithm of mobile geographical information.

\section{DisPlAY CONTROL ALGORITHM}

It consumes much memory to current architectures and systems used in the portable equipment, and it even does not allow managing greater image data. [8] To allow a big image data to be used in the portable equipment, an effective display control algorithm is needed.

There are three steps to achieve the aim of display control.

First, the user's state such as situation is detected by mobile support station (MSS) to get the detailed moving parameter when the user sends out querying requirement of geographical information, and then, the result of measurement is transformed into the standard format of mobile GIS.

Second, the transformed result of measurement is regarded as condition to query database of mobile GIS. If some geographical information object or a part of it need be displayed on the screen, the geographical information object will be downloaded and saved in portable equipment memory so as to process it in the next step.

At last, display control technique is used to cut down the needless display parts in the querying result image, so the image that will be drawn is just the parts, which could be 
shown in the screen. In order to meet the requirement, we design a display control algorithm. The process is: First, get the points at which the extending lines of four edges of the rectangular plane limited by the sides of the portable equipment screen intersect with the geographical information object, and insert the points into a singly linked lists in serialization order; And then the points lying on the extending lines of four edges of the rectangular plane limited by the sides of the portable equipment screen are moved to the tops of the angle of the rectangular plane limited by screen; Last, the points lying out of the rectangular plane limited by screen are deleted. During the process, if any two neighboring points have the same value, they should be combined into one point. Using the algorithm, we could realize the display control technique of mobile geographical information based on measure of user's location.

In order to explain the algorithm in detail, we put forward some definitions and regulations.

Definition 1: The point lying on the sides of screen or out of screen is the out point.

Definition 2: The point lying on the sides of screen or in the screen is the useful point.

Definition 3: The point, which has no use for drawing the result image in the screen, is the useless point.

Regulation 1: The useless points decision regulation

- If there are three out points to be connected together and the middle one is not useful point, then the middle point is the useless point;

- If both the beginning point and the next point are out points, and the beginning point is the useless point, the beginning point is the useless point;

- If both the ending point and the previous point are out points, and the ending point is the useless point, the ending point is the useless point;

Regulation 2: The adding points regulation

Let the rectangular plane limited by screen be rectangle $\mathrm{A}$, and let the needed display object be polygon $\mathrm{B}$. Let the four edges of the rectangle A lie on straight line a, b, c, d separately. Get the points at which straight lines a, b, c, d intersect with polygon $\mathrm{B}$, and then insert them into the linked lists at corresponding positions.

Regulation 3: The moving points regulation

If the obtained point through the adding points regulation lies on the extending line of four edges of the rectangular plane limited by screen, the point should be moved to the vertex of the screen, which is nearest to the point.

Regulation 4: The combining points regulation

If the neighboring two points in the linked lists have the same value, they should be combined to one point.

Regulation 5: The deleting points regulation

Use the useless points decision regulation to find the useless points in the linked lists, and delete them from the linked lists.

\section{EXPLANATION AND EXAMPLE}

Next, we are explaining how to control the display of geographical information objects using the given definition and regulation above. Suppose that the polygon ABCDEF is a geographical information object, which need be displayed on the screen, and the rectangle A'B'C' $D$ ' is the control rectangle limited by screen. Let edge $A^{\prime} B^{\prime}$ lie on straight line $a$, edge $B$ ' $C$ ' lie on straight line $b$, edge C'D' lie on straight line c, and edge D'A' lie on straight line d.

At the beginning, according to Figure 1, we know that, the original linked lists is:

$$
\mathrm{A} \rightarrow \mathrm{B} \rightarrow \mathrm{C} \rightarrow \mathrm{D} \rightarrow \mathrm{E} \rightarrow \mathrm{F} \rightarrow \mathrm{A} ;
$$

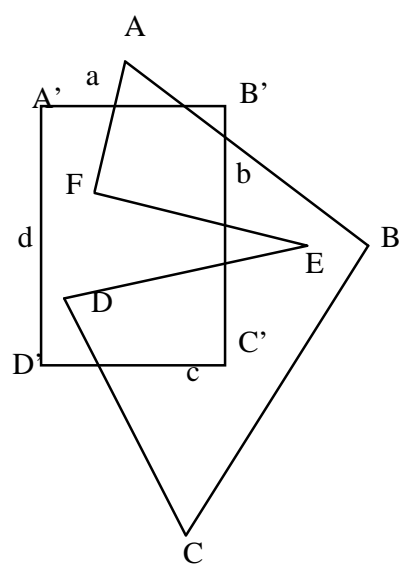

Figure 1. The original picture

If we want to show geographical information object ABCDEF by linked lists method. Obviously, we need the display control technique, which we talk about above. In an other word, we should get the part of geographical information object ABCDEF showing in the screen A'B'C'D'. These steps could make the aim become reality:

Step 1: Use the adding points regulation to seek the points at which the lines a, b, c, d intersect with the geographical information object $\mathrm{ABCDEF}$, and then insert them into the linked lists. The process is detailed below:

- At first, the points at which the line a intersect with segments of line $\mathrm{AB}, \mathrm{BC}, \mathrm{CD}, \mathrm{DE}, \mathrm{EF}, \mathrm{FA}$ need be gotten: By calculating, line a intersects with segment of line $A B$ at point 2, and line a intersects with segment of line FA at point 1 ; There are no joints between line a and other segments of line, so we don't calculate them. Because of point 2 lying between point $A$ and point $B$, point 2 is inserted into linked lists between point $\mathrm{A}$ and point $\mathrm{B}$. The same as point 2 , point 1 need be inserted into linked lists between point $\mathrm{F}$ and point $\mathrm{A}$. Now, the linked lists turns into:

$\mathrm{A} \rightarrow 2 \rightarrow \mathrm{B} \rightarrow \mathrm{C} \rightarrow \mathrm{D} \rightarrow \mathrm{E} \rightarrow \mathrm{F} \rightarrow 1 \rightarrow \mathrm{A} ;$

- Next, get the points at which the line b intersects with segments of line A2, 2B, BC, CD, DE, EF, F1, $1 \mathrm{~A}$ and get the joints: point 5 , point 6 , point 7 , point 8 , and insert them into the linked lists. Now, the linked lists turns into: 
$\mathrm{A} \rightarrow 2 \rightarrow 3 \rightarrow \mathrm{B} \rightarrow 6 \rightarrow \mathrm{C} \rightarrow \mathrm{D} \rightarrow 5 \rightarrow \mathrm{E} \rightarrow 4 \rightarrow \mathrm{F} \rightarrow 1 \rightarrow \mathrm{A} ;$

- Then, get the points at which the line c intersects with segments of line A2, 23, 3B, B6, 6C, CD, D5, $5 \mathrm{E}, \mathrm{E} 4,4 \mathrm{~F}, \mathrm{~F} 1,1 \mathrm{~A}$ and get the joints: point 5 , point 6 , point 7 , point 8 , and insert them into the linked lists. Now, the linked lists turns into:

$\mathrm{A} \rightarrow 2 \rightarrow 3 \rightarrow \mathrm{B} \rightarrow 7 \rightarrow 6 \rightarrow \mathrm{C} \rightarrow 8 \rightarrow \mathrm{D} \rightarrow 5 \rightarrow \mathrm{E} \rightarrow 4 \rightarrow \mathrm{F} \rightarrow 1$ $\rightarrow \mathrm{A}$;

- At last, seek the points at which the line d intersects with segments of line A2, 23, 3B, B7, 76, 6C, C8, 8D, D5, 5E, E4, 4F, F1, 1A. By calculating, there are no joints between line $d$ and these segments of line, so the linked lists need not change.

The Figure 2 shows the result after adding points.

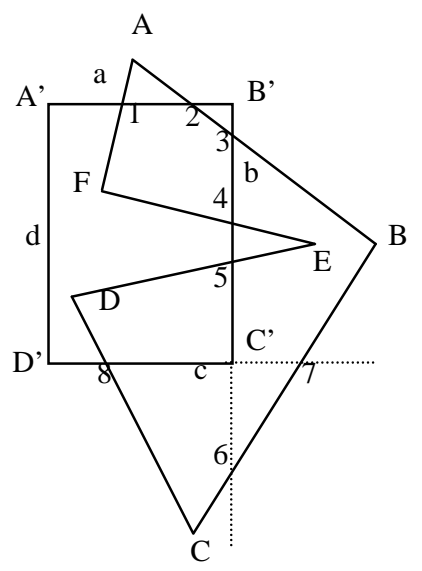

Figure 2. The result after adding points

Step 2: Using the moving points regulation, let point 7 and point 6 which are added into the linked lists in previous step move to point C'. Then the linked lists becomes: A;

$$
\mathrm{A} \rightarrow 2 \rightarrow 3 \rightarrow \mathrm{B} \rightarrow \mathrm{C}^{\prime} \rightarrow \mathrm{C}^{\prime} \rightarrow \mathrm{C} \rightarrow 8 \rightarrow \mathrm{D} \rightarrow 5 \rightarrow \mathrm{E} \rightarrow 4 \rightarrow \mathrm{F} \rightarrow 1 \rightarrow
$$

Step 3: Use the combining points regulation to simplify the linked lists. Now the linked lists turns into:

$$
\begin{aligned}
& \mathrm{A} \rightarrow 2 \rightarrow 3 \rightarrow \mathrm{B} \rightarrow \mathrm{C}^{\prime}(6,7) \rightarrow \mathrm{C} \rightarrow 8 \rightarrow \mathrm{D} \rightarrow 5 \rightarrow \mathrm{E} \rightarrow 4 \rightarrow \mathrm{F} \rightarrow 1 \\
& \rightarrow \mathrm{A} ;
\end{aligned}
$$

The Figure 3 shows the result after combination.
A

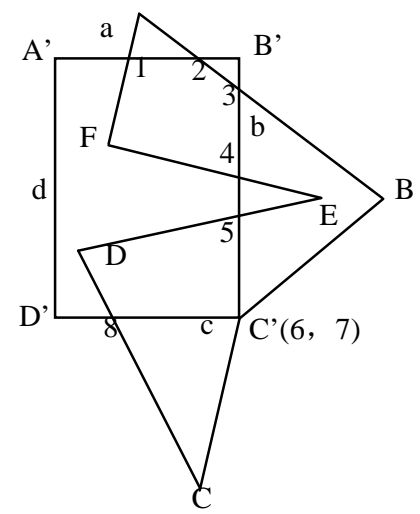

Figure 3. The result after moving points and combining point

Step 4: Use the useless points decision regulation and the deleting points regulation to delete the useless points from the linked lists. The process is detailed below:

- According to definition 1, we know that, point A and point 2 are out points. And according to the useless points decision regulation (b), the beginning point $\mathrm{A}$ could be deleted. Using the deleting points regulation, the linked lists turns into:

$2 \rightarrow 3 \rightarrow B \rightarrow C^{\prime} \rightarrow C \rightarrow 8 \rightarrow D \rightarrow 5 \rightarrow E \rightarrow 4 \rightarrow F \rightarrow 1 \rightarrow A ;$

- $\quad$ Since point 3 , point $B$, point $C^{\prime}$ are out points, point $\mathrm{B}$ could be deleted according to the useless points decision regulation (a). Point $\mathrm{C}$, point $\mathrm{E}$ could be deleted the same as point $\mathrm{C}$. Using the deleting points regulation, now the linked lists turns into: $2 \rightarrow 3 \rightarrow C^{\prime} \rightarrow 8 \rightarrow D \rightarrow 5 \rightarrow 4 \rightarrow F \rightarrow 1 \rightarrow A$;

- According to definition 1 , point 1 and point A are out points, and according to the useless points decision regulation (c), the ending point A could be deleted. Using the deleting points regulation, the linked lists finally turns into:

$2 \rightarrow 3 \rightarrow \mathrm{C}^{\prime} \rightarrow 8 \rightarrow \mathrm{D} \rightarrow 5 \rightarrow 4 \rightarrow \mathrm{F} \rightarrow 1$;

The Figure 4 shows the final result.

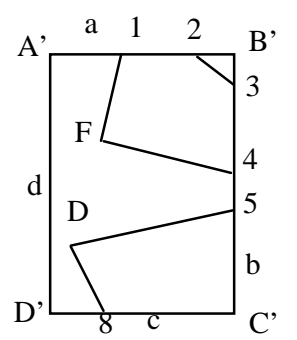

Figure 4. The final result

The vectorgraph decided by points $2,3, C^{\prime}, 8, \mathrm{D}, 5,4, \mathrm{~F}$, 1 in serialization order is the querying result image, and its 
effect in the screen is equal to the effect showed by polygon ABCDEF, but the needed display memory is lower.

\section{EXPERIMENT}

The principles described in this paper are derived from the experiences and outcomes of various real world projects. So we have examined the efficiency of the display control algorithm, and designed an experiment on Android operating system. Android operating system is a Linux-based operating system designed primarily for touch screen mobile devices such as smart phones and tablet computers. It is currently developed by Google, and it's a good platform to develop and test new applications. Using the Android software development kit, we can design and develop any new applications we need, and the new applicants also can be implanted into any portable equipment with Android operating system. So it is fit for examining our algorithm.

In our experiment, we send out a requirement with portable equipment that is implanted the Android platform and running our Mobile GIS with display control technique. During the system running, we check the usage of memory, processor, display memory, and find out they all have more efficient than the GIS without our display control algorithm. Figure 5 compares the mean time of showing the querying result image between with our display control technique and without our display control technique on Android platform.

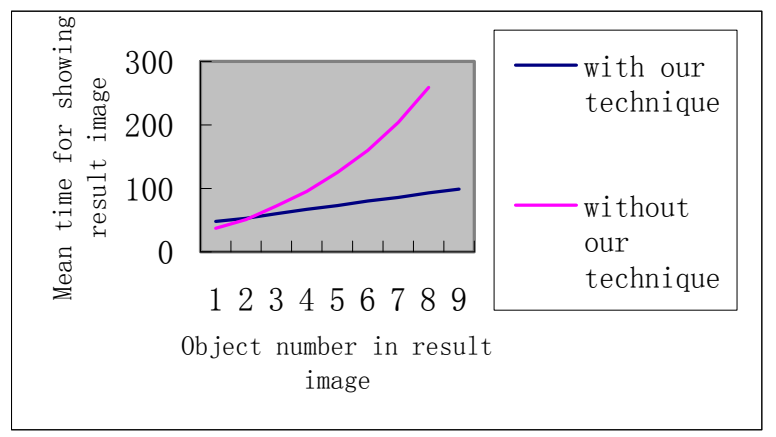

Figure 5. Compare the mean time for showing image

\section{EXPERIMENT}

Big Data is a new label given to a diverse field of data intensive informatics in which the data sets are so large that they become hard to work with effectively. This paper discusses how the user could gain awareness of the personally relevant part big image data that is publicly available in the portable equipment with mobile GIS. At the same time, this paper puts forward a display control technique on portable equipment, which is based on measurement of user's location. A simulation experiment has been done on Android platform, and the result shows that the performance of portable equipment with display control technique is higher than the portable equipment without display control technique. The expected goal has been achieved.

\section{ACKNOWLEDGMENT}

This paper is supported by the Fundamental Research Funds for the Central Universities (No.2012XJ031). Without this help, this work would never have been completed.

\section{REFERENCES}

[1] Qin Jingtao, and Wu Sheng, "Studies on multi-source image data management based on GeoRaster”, 2010 Second IITA International Conference on Geoscience and Remote Sensing (IITA-GRS 2010), 2010, pp.487-491, doi: 10.1109/IITA-GRS.2010.5602755.

[2] A. Fiolek, and D.W. Collins, "Video Data Management System archives and provides online access to NOAA deep-sea corals digital video and image data”, OCEANS 2008, 2008, pp.1-6, doi: 10.1109/OCEANS.2008.5151940.

[3] Joo-Young Kim, Donghyun Kim, Seungjin Lee, Kwanho Kim, and Hoi-Jun Yoo, "Visual Image Processing RAM: Memory Architecture With 2-D Data Location Search and Data Consistency Management for a Multicore Object Recognition Processor”, IEEE Transactions on Circuits and Systems for Video Technology, 2010, 20(4), pp.485-495, doi: 10.1109/TCSVT.2009.2035830.

[4] Yu Liu, Kexiong Chen, Feng Gu, Jicheng Quan, and Guang Yu, "Research and implementation of mass remote sensing image data storage and management", 2010 IEEE International Conference on Progress in Informatics and Computing (PIC 2010), 2010, pp.609-612, doi: 10.1109/PIC.2010.5687478.

[5] K. Iwamura, K. Muro, N. Ishimaru, and M. Fukushima, "4D-GIS (4 dimensional GIS) as spatial-temporal data mining platform and its application to managementand monitoring of large-scale infrastructures”, 2011 IEEE International Conference on Spatial Data Mining and Geographical Knowledge Services (ICSDM 2011), 2011, pp.38-43, doi: 10.1109/ICSDM.2011.5969001.

[6] Run-sheng Li, Shu-long Zhu, Wei-can Meng, and Wen Cao, "Data Organization and Management of Image Navigation”, 2010 Second World Congress on Software Engineering (WCSE 2010), 2010, pp.22-25, doi: 10.1109/WCSE.2010.74.

[7] I.G. Olaizola, N. Aginako, and M. Labayen, "Image Analysis Platform for Data Management in the Meteorological Domain", 4th International Workshop on Semantic Media Adaptation and Personalization (SMAP 2009), 2009, pp.89-94, doi: 10.1109/ SMAP.2009.29.

[8] Z. Larabi, Y. Mathieu, and S. Mancini, "Efficient Data Access Management for FPGA-Based Image Processing SoCs", IEEE/IFIP International Symposium on Rapid System Prototyping(RSP 2009), 2009, pp.159-165, doi: 10.1109/RSP.2009.20. 